\title{
Numerical Modeling of MEMS Structures Involving Motion Effected by the Coupling of Maxwell's and Mechanical Equations
}

\author{
Kohei Kawano $^{(1)}$ Takashi Mori $^{(1)}$ Michiko Kuroda $^{(1)}$ \\ Manos M. Tentzeris ${ }^{(2)}$ \\ kuroda@cc.teu.ac.jp \\ ${ }^{(1)}$ School of Engineering, Tokyo University of Technology, Hachioji, Tokyo 192-0982, Japan \\ ${ }^{(2)}$ School of ECE, Georgia Institute of Technology, Atlanta, GA 30332-250, USA

\begin{abstract}
The adaptive body fitted grid generation method for moving boundaries is applied to the analysis of MEMS-based variable devices with the combined effect of mechanical and electrical forces. MEMS technology is growing rapidly in the RF field and the accurate design of RF MEMS structures that can be used for phase shifting or reconfigurable tuners requires the computationally effective modeling of their transient and steady-state behavior including the accurate analysis of their time-dependent moving boundaries. The technique proposed in this paper is based on the finite-difference time-domain method with an adaptive implementation of grid generation. In this paper, this simulation method is applied to the analysis of a two-dimensional MEMS variable capacitor with non-uniform motions such as accelerated motions. The acceleration of the MEMS capacitor is derived under the equilibrium between the spring force and electrical force. By substituting this acceleration value into the transformation function, the relation between the capacitance and the motion characteristics is derived. The relation between the bias voltage and the displacement is shown.
\end{abstract}

\section{Introduction}

RF MEMS technology has been developed rapidly, because of the advantage over p-i-n diode or FET switches[1],[2]. An accurate knowledge of the electromagnetic field evolution around a moving or rotating body is required for the realization of new optical devices and microwave devices, such as the RF-MEMS structures used in phase-shifters, couplers or filters. Computational technique for the moving boundary problems have been pursued mainly in heat and fluid flow area [3],[4]. In this paper, we propose a new numerical approach for the analysis of this type of problems that alleviates the limitations of the conventional time-domain techniques [5],[6]. By using a transformation with a time factor, it is possible to apply the grid generation technique of [7] to the time-domain analysis of the moving object. It is a feature that makes the new method especially useful for analyzing moving objects of arbitrary shape. In this paper, this simulation method is applied to the analysis of a two-dimensional MEMS variable capacitor with accelerated motion derived from the coupling of the mechanical and Maxwell equations.

\section{Two- Dimensional Variable Capacitor with Accelerated Motion Effect}

The geometry considered here is shown in Fig.1. Under the combined effect of mechanical and electrical force, the two plates are assumed to move for $x$-direction. In the case of two-dimensional TM-propagation, as shown in Fig.1, the only nonzero components are $E x, E y, H z$, and they exhibit a time evolution expressed by:

$$
\begin{gathered}
\frac{\partial H_{z}}{\partial t}=\frac{1}{\mu}\left(\frac{\partial E_{x}}{\partial y}-\frac{\partial E_{y}}{\partial x}\right) \\
\frac{\partial E_{x}}{\partial t}=\frac{1}{\varepsilon}\left(\frac{\partial H_{z}}{\partial y}-J_{x}\right)
\end{gathered}
$$




$$
\frac{\partial E_{y}}{\partial t}=-\frac{1}{\varepsilon}\left(\frac{\partial H_{z}}{\partial x}+J_{y}\right)
$$

where $\varepsilon, \mu$ are the constitutive parameters of respective medium. Under the combined effect of mechanical and electrical force, the plate moves until the equilibrium between the electrostatic and mechanical forces is reached. These forces are expressed in the following equations, respectively.

$$
\begin{aligned}
& F_{m}=m x^{\prime \prime}+b x^{\prime}+k x \\
& F_{e}=\frac{1}{2} \frac{\partial C}{\partial x} V^{2}
\end{aligned}
$$

, where $F m$ means spring force, $F e$ means electrostatic force, $m$ is the mass of the plate, $b$ is the mechanical resistance, $k$ is the spring constant and $V$ is the bias voltage. From the equilibrium between spring force and electrostatic force, eq.(6) is derived.

$$
m x^{\prime \prime}=\frac{1}{2} \frac{\partial C}{\partial x} V^{2}-b x^{\prime}-k x
$$

Then, from eq.(6), the acceleration $x "$ is obtained. In Fig.1, the configurations of the physical and of the computational regions are shown. Under the combined effect of mechanical and electrical force, the plates are assumed to move for $x$-direction with velocities $v$ and $u$, and the acceleration $a_{v}, a_{u}$ respectively. Using a coordinates' transformation technique, the time-changing physical region $(x, y, t)$ can evolve to a time-invariant computational domain. For the geometry of Fig.1, the transform equations between the physical and the computational regions are chosen as :

$$
\begin{aligned}
& \xi=\frac{x-h_{n}(t)}{h_{n+1}(t)-h_{n}(t)} \\
& \eta=\frac{y-y_{m}(t)}{y_{m+1}(t)-y_{m}(t)} \\
& \tau=t
\end{aligned}
$$

,where $\mathrm{n}=1,2,3, \mathrm{~m}=1,2,3$ and $h_{1}(t), h_{2}(t), h_{3}(t), h_{4}(t)$, are written in the following form assuming that the plate velocities remain time changing values for the whole time of their motion.
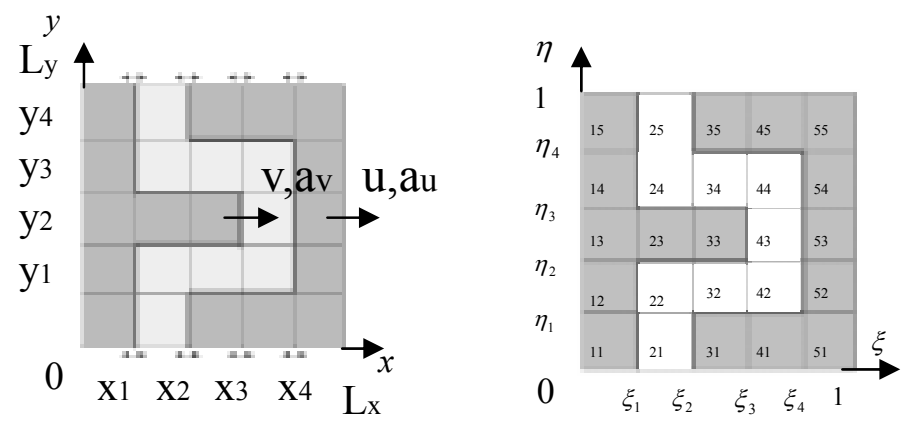

Fig1. Physical region and computational region 


$$
\begin{aligned}
& h_{1}(t)=x_{1}+v(t) t+\frac{1}{2} a_{v}(t) t^{2} \\
& h_{2}(t)=x_{2}+u(t) t+\frac{1}{2} a_{u}(t) t^{2} \\
& h_{3}(t)=x_{3}+v(t) t+\frac{1}{2} a_{v}(t) t^{2} \\
& h_{4}(t)=x_{4}+u(t) t+\frac{1}{2} a_{u}(t) t^{2}
\end{aligned}
$$

The functions $h_{1}(t), h_{2}(t), h_{3}(t), h_{4}(t)$,describe the movement along the $x$ axis, and allow for the realization of a rectangular grid with stationary boundary conditions. The partial time-derivatives in the transformed domain $(\xi, \eta, \tau)$ can be expressed in terms of the partial derivatives of the original domain $(x, y, t)$ using eqs.(7)-(13). The FDTD technique can provide the time-domain solution of the rectangular $(\xi, \eta, \tau)$ grid. The stability criterion in this case is chosen as $c \Delta t \leq \delta / \sqrt{2}$, where $\delta=\Delta x_{0}=\Delta y_{0}$, assuming the grid is uniformly discretized in both directions. In general, $\delta$ is a space increment for $x$ and $y$ direction when the grid increment is minimum (minimum cell size).

\section{Numerical Results}

To validate this approach, numerical results are calculated for a two-dimensional variable capacitor with the movement of the finger only to the $x$-direction. The grid includes $200 \times 200$ cells and, $L_{x}=L_{y}=L_{z}=L=5 \lambda, \Delta x=\Delta y=L / 200, \Delta t=L / 800 c$. The initial plate separation is $L_{x} / 5$, the area is $S=L_{y} / 5 \times L_{z}$ and the grid is terminated with Mur's absorbing boundary conditions. Here, the left plate is assumed to move due to the coupling of the electrostatic and the mechanical forces. Fig.2 displays the computational algorithm used in this calculation. Initial values of the velocity are assumed $u_{0}=0, v_{0}=0$ and $a_{u}=0 . a_{v}=0$. From eq.(6), acceleration value is derived. By substituting this acceleration value into the transform function $h_{1}(t), h_{3}(t)$, new capacitance $C$ and displacement $h_{1}(t), h_{3}(t)$ and acceleration $a_{v}$ are obtained. And then from the capacitance and displacement, new acceleration is obtained. Iterating of this algorithm, it is easy to get the capacitance, acceleration and displacement controlled by the coupling of spring and electrostatic force.

$$
\begin{gathered}
v_{0}=u_{0}=0, a_{u}=0, a_{v}=0 \\
a_{v}=x "=\frac{1}{m}\left(-k\left(h_{3}(t)-x_{3}\right)+\frac{1}{2} \frac{\partial C}{\partial x} V^{2}\right) \\
\Delta d(t)=\frac{1}{2} a_{v}(t) \Delta t^{2}+v(t) \Delta t \\
h_{3}(t)=\sum_{t=1}^{T} \Delta d(t)+x_{3}
\end{gathered}
$$

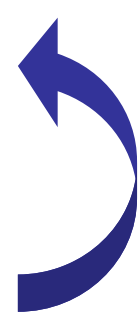

Fig2 Computational Algorithm 
Numerical results are shown in Fig.3, where $\mathrm{f}=20 \mathrm{GHz}, k=10^{5} \mathrm{~N} / \mathrm{m}, m=10^{-7} \mathrm{~kg}, b=0$. In Fig.3 tuning characteristics between displacement and bias voltage are shown. The displacement increases gradually with bias voltage.

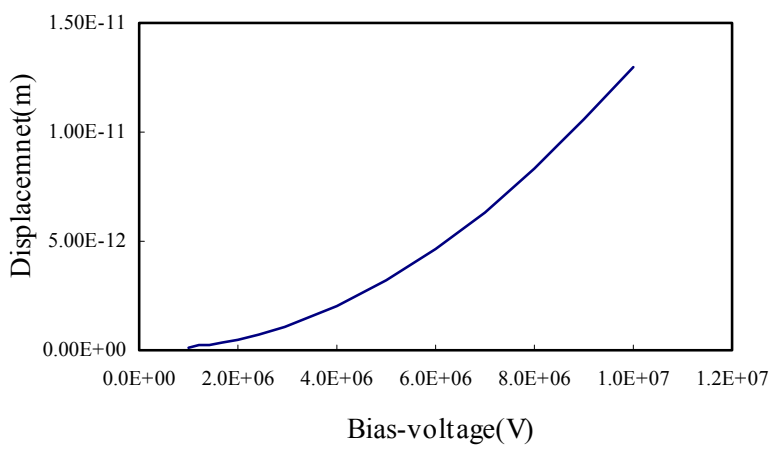

Fig3 Bias-Voltage vs displacement

$(\mathrm{t}=1200$ time-step $)$

\section{Conclusions}

The modeling technique of the transient effect of MEMS structures with accelerated motion is proposed. This technique is a combination of the FDTD method and the body fitted grid generation technique. The acceleration value is derived by the coupling of combined effect of electrical and mechanical forces. The numerical results of the relation between the capacitance and the displacement is shown for a MEMS capacitor and demonstrate its unique computational advantages in the modeling of microwave devices and/or optical devices with moving boundaries.

\section{Acknowledgement}

The authors wish to acknowledge the support of Garnt-in-Aid for Scientific Research ((c) (2) No.15560309) of The inistry of Education, Culture, Sports, Science and Technology(MEXT),Japan and Yamacraw Design Center of the State of Georgia, the NSF Career Award under contract NSF \#9964761, and the NSF Packaging Research Center of Georgia Tech

\section{Reference}

[1]Aleksander Dec, Ken Suyama ,'Microwave MEMS-Based Voltage-Controlled Oscillators, IEEE Trans MTT, pp.1943-1949, vol.48, No.11, Nov.2000.

[2]N. Bushvager , B. McGarvey, M. Tentzeris, ”Adaptive Numerical Modeling of RF Structures requiring the Coupling of Maxwell's mechanical and Solid-State Equations", Proc. of ACES 2001, pp.1-6, March 2001, Monterey, CA..

[3]S. Kuroda, H. Ohba, "Numerical analysis of flow around a rotating square cylinder", JSME International Journal, 36-4 B, pp.592-597, 1993.

[4]P. J. Zwart, G. D.Raithby, "Space-time meshing for two dimensional moving boundary problems", Proc. of the $7^{\text {th }}$ International Meshing Roundtable, Oct., 1998. Dearborn, Mich.

[5]M. Kuroda, "Electromagnetic wave scattering from perfectly conducting moving boundary- An application of the body fitted grid generation with moving boundary", IEICE Trans, Vol.E77-C, No.11, pp.1735-1739, Nov. 1994.

[6] M. Kuroda, N. Miura, M.M.Tentzeris, "A Novel Numerical Approach for the Analysis of 2D MEMS-Based Variable Capacitors with Arbitrary Motions" Proc. of ACES2003, pp48-53, Monterey March, 2003

[7] J.F.Thompson, "Numerical Grid Generation ”, North Holland, Amsterdam, 1985.

[8] V. Bladel, "Relativity and Engineering", Springer-Verg, Berlin, 1984. 